\title{
Association of Neutrophil Gelatinase associated Lipocalin and Leukocyte Differential Count in Children with Febrile Urinary Tract Infections
}

\author{
Ji Won Jang, M.D. \\ Hyung Eun Yim, M.D., Ph.D. \\ Kee Hwan Yoo, M.D., Ph.D. \\ Department of Pediatrics, Korea \\ University College of Medicine, Seoul, \\ Korea
}

Corresponding author:

Hyung Eun Yim, M.D., Ph.D.

Department of Pediatrics, Korea University

Ansan Hospital, Korea University College

of Medicine, 123 Jeokgeum-ro, Danwon-

gu, Ansan 15355, Republic of Korea

Tel: +82-31-412-5096

Fax: +82-31-405-8591

E-mail: he-yim@hanmail.net

Received: 16 June 2020

Revised: 17 September 2020

Accepted: 21 September 2020
Purpose: To investigate the association between urinary neutrophil gelatinaseassociated lipocalin (UNGAL) and leukocyte differential count in children with urinary tract infections (UTIs).

Methods: A retrospective chart review was performed in children undergoing uNGAL measurements between June 2018 and September 2019. Patients with suspected or diagnosed UTIs were included. The relationship between UNGAL and blood leukocyte differential count was investigated in children.

Results: A total of 197 children were included in this study, 119 of whom (60\%) had UTIs. The non-UTI patients $(\mathrm{n}=78)$ were diagnosed with pneumonia, acute gastroenteritis, viral upper respiratory infection, and others. After adjusting for age, gender, and fever duration, the leukocyte count, monocyte count, and uNGAL levels were higher in the UTI group than in the non-UTI group $(P<0.05)$. UNGAL showed positive correlations with neutrophil counts, monocyte counts, the neutrophil-to-lymphocyte ratio, and the monocyte-to-lymphocyte ratio in the UTI group $(P<0.05)$. UNGAL levels were only associated with the neutrophil-tolymphocyte ratio in the non-UTI group $(P<0.05)$. In a multivariable logistic regression analysis, only uNGAL was associated with the presence of UTI $(P<0.05)$. The area under the receiver operating characteristic curves for UNGAL and monocyte counts to identify UTI were 0.89 (95\% confidence interval (Cl): 0.824-0.939; $P=$ 0.025 ) and 0.7 ( $95 \% \mathrm{Cl}: 0.627-0.774 ; P=0.038)$, respectively.

Conclusions: In children with UTIs, UNGAL levels may be associated with blood leukocyte differential counts. UNGAL measurements and monocyte counts can be helpful in children with suspected UTIs.

Key words: Biomarkers, Monocytes, Urinary tract infections

\section{Introduction}

Urinary tract infection (UTI) is one of the most common febrile infectious

This is an open-access article distributed under the terms of the Creative Commons Attribution Non-Commercial License (http:// creativecommons.org/licenses/by-nc/4.0/) which permits unrestricted non-commercial use, distribution, and reproduction in any medium, provided the original work is properly cited. diseases in children ${ }^{1)}$. Early detection of UTI and proper management are critical since UTI can lead to permanent kidney damage, hypertension, chronic kidney disease (CKD), and ultimately end-stage renal disease ${ }^{2}$. However, UTIs are not easy to recognize because the symptoms and signs may be ambiguous, especially in young children ${ }^{3)}$. Besides, there are some other difficulties in diagnosing UTI in young children, such as delayed urine sampling and frequent over-diagnosis from contaminated urine specimens ${ }^{4}$. There is 
also a practical difficulty in accurately interpreting the number of bacteria in the urine, and distinguishing between asymptomatic bacteriuria and a true $\mathrm{UTI}^{5,6}$. Therefore, other markers for diagnosing UTI are needed. And several studies for new markers such as procalcitonin and leukocyte esterase have been conducted recently ${ }^{4,7)}$.

Neutrophil gelatinase-related lipocalin (NGAL) is a member of the lipocalin superfamily ${ }^{8)}$. It is a $25-\mathrm{kDa}$ protein covalently linked to gelatinase in human neutrophils and highly expressed in neutrophils, monocytes, and macrophages $^{8)}$. As an iron carrier protein, NGAL plays a role in innate immunity. It binds bacterial siderophores and thus, limits bacterial growth ${ }^{9)}$. It has been reported that NGAL could be a useful predictor for differentiating acute bacterial infections from viral ones during acute infections in children ${ }^{10}$. Several studies have also demonstrated the diagnostic utility of urinary and plasma NGAL in detecting UTIs experimentally and clinically ${ }^{8,11,12)}$.

Leukocyte differential counts have long been used to determine the cause of infection ${ }^{13-17)}$. When an infection occurs, neutrophils and monocytes move to the peripheral site, which is essential for defending against pathogens ${ }^{18}$. As the infection progresses, the number of neutrophils and monocytes increases rapidly, and the cells migrate to the inflamed tissue ${ }^{18)}$. Several studies have revealed a link between monocytes, infections, and immune mechanisms ${ }^{19-}$ ${ }^{21)}$. Another paper reported that neutrophils and monocytes had an effect on combating microbial pathogens ${ }^{22)}$. Monocytes defend bacteria by providing macrophages and dendritic cell precursors to tissues ${ }^{22}$. A previous study found that the neutrophil-to-lymphocyte ratio (NLR) and monocyte-to-lymphocyte ratio (MLR) may help in determining which of the patients with fever are more likely to have bacterial infections and should be considered for antibiotic treatment ${ }^{13)}$.

Considering these facts, we hypothesized that NGAL relates to leukocyte differential count in blood in children with UTIs. Therefore, in this study, we aimed to investigate the association between urinary NGAL (uNGAL) and leukocyte differential counts in children with febrile UTIs. The diagnostic accuracy of uNGAL and leukocyte differential counts for identifying UTIs was also examined.

\section{Materials and methods}

\section{Patients and inclusion criteria}

A retrospective chart review was performed in children undergoing urinary NGAL measurements between June 2018 and September 2019. The data from 417 pediatric patients with suspected UTIs who were admitted to the Korea University Guro Hospital were included. And the age of the patient was composed from minimum 1 month to maximum 12 years. The diagnosis of first febrile UTI was as follows : (1) fever of $\geq 38^{\circ} \mathrm{C}$, (2) pyuria ( $\geq 5$ white blood cells (WBC)s per high-power field), (3) positive urine culture collected from a midstream urine (defined as the growth of a single organism with $\geq 100,000$ colony-forming units/ $\mathrm{mL}$ or $\geq 50,000$ colony-forming units $/ \mathrm{mL}$ with clinical sign of infection such as fever) or catheterized specimen (defined as the growth of a single organism with $\geq 50,000$ colonyforming units $/ \mathrm{mL})^{23)}$ and (4) no previous history of UTI. Patients with recurrent UTIs, acute kidney injury (AKI), CKD, systemic disease, and known congenital anomalies of the kidney and the urinary tract (CAKUT) were excluded.

\section{Measurements}

First, we investigated children who were tested for UNGAL for suspected UTI between June 2018 and September 2019. A chart review of children who met the inclusion criteria was conducted retrospectively in the electronic medical records. The children were divided into the UTI group and the non-UTI group. Clinical data, such as age, sex, and duration of fever were collected. Also, the results of blood tests performed prior to the initiation of antibiotic treatment, including complete blood cell count ( $\mathrm{CBC}$ ), leukocyte, neutrophils, lymphocytes, monocyte counts, and C-reactive protein (CRP) results were collected. The NLR and MLR were then calculated using the neutrophil, lymphocyte, and monocyte counts. Urine samples for urinalysis, urine culture, and the uNGAL test were also collected before antibiotic treatment. uNGAL test was performed using the ARCHITECT Urine NGAL assay. It uses NGAL mouse monoclonal anti-NGAL antibody-coated microparticles and arcidine-related anti-NGAL antibodies ${ }^{24)}$. Clinical data including age, sex, and fever duration, and laboratory data including uNGAL measurement, leukocyte count, neutrophil count, and monocyte count were compared 
between the UTI and non-UTI groups. The association between UNGAL and the leukocyte differential count in children with or without febrile UTI was investigated. The relationship between serum CRP levels and the leukocyte differential count was additionally examined.

\section{Statistics}

IBM SPSS ver. 20.0 (IBM Co., Armonk, NY, USA) was used to compare the variables. The continuous variables are presented as mean \pm standard deviation or standard error of the mean and the categorical variables as number (\%). The Mann-Whitney U test was used for continuous variables, such as age, leukocyte count, absolute neutrophil count (ANC), lymphocyte count, monocyte count, NLR, MLR, and UNGAL. The Chi-squared test was used for categorical variables such as gender. To control for the effects of age, gender, and fever duration, analysis of covariance (ANCOVA) was used. Univariable and multivariable logistic regression analyses were also performed to find the predictive factors for UTI. The odds ratio (OR) of each variable for UTI was calculated using logistic regression analysis. Variables with a $P$-value of $\leq 0.20$ in univariable analyses were included in the multivariable model. Correlation analysis was carried out to determine the relationship between two variables by Spearman's correlation test. The discriminative ability of each biomarker for UTI was evaluated by plotting ROC curves for the biomarkers. The area under the ROC curve (AUC) with a 95\% confidence interval (CI) was calculated and compared between the variables. All differences were considered significant at a $P$-value of $<0.05$.

\section{Results}

\section{Patient characteristics}

Among 417 children with suspected UTIs, a total of 197 patients were included in this study. One hundred nineteen patients were diagnosed with UTIs. The patients with nonUTI $(n=78)$ were diagnosed with pneumonia $(n=18)$, acute gastroenteritis $(n=12)$, viral upper respiratory infection $(n=30)$, and others ( $n=18)$ (Fig. 1). The proportion of female patients was higher in the non-UTI group than in the UTI group $(P<0.05)$. However, there were no differences in age and fever duration (>72 hours) between the two groups. Children with UTIs showed higher monocyte counts, uNGAL levels, and blood urea nitrogen (BUN)-to-creatinine $(\mathrm{Cr})$ ratios compared to the non-UTI group $(P<0.05)$. Serum Cr concentration, WBC count, and CRP level were not different between the two groups (Table 1). When the age, gender, and fever duration-adjusted predicted means were compared between the UTI and non-UTI groups,

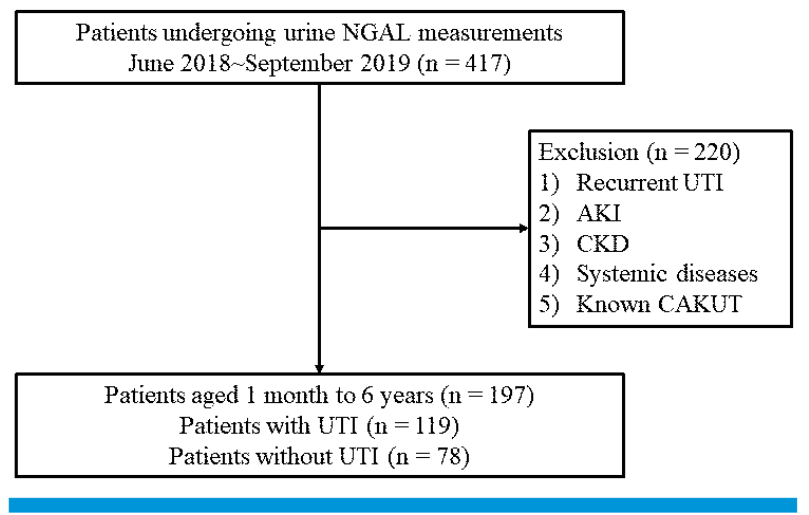

Fig. 1. Study flow diagram. NGAL, urinary neutrophil gelatinaseassociated lipocalin.

UTI, Urinary tract infection; AKI, acute kidney injury; CKD, chronic kidney disease; CAKUT, congenital anomalies of the kidney and urinary tract.

Table 1. Patient Characteristics and Clinical Findings between the UTI and Non-UTI Groups

\begin{tabular}{|c|c|c|c|}
\hline & Non-UTI & UTI & $P$-value \\
\hline Age (month) & $32.5 \pm 30.6$ & $20.1 \pm 41.0$ & $0.774^{*}$ \\
\hline Female, n (\%) & 45/78 (58\%) & $40 / 119$ (34\%) & $0.024^{\dagger}$ \\
\hline \multicolumn{4}{|l|}{ Fever duration } \\
\hline Total (>72 hrs), n (\%) & 25/78 (32.1\%) & $31 / 119(26.1 \%)$ & $0.079^{\dagger}$ \\
\hline WBC count $\left(/ \mathrm{mm}^{3}\right)$ & $11,073 \pm 6,110$ & $13,517 \pm 5,024$ & $0.36^{*}$ \\
\hline ANC & $6,124 \pm 5,157$ & $7,089 \pm 4,110$ & $0.146^{*}$ \\
\hline Lymphocyte count (/mm³) & $3,857 \pm 2,366$ & $4,706 \pm 2,378$ & $0.867^{*}$ \\
\hline Monocyte count $\left(/ \mathrm{mm}^{3}\right)$ & $953 \pm 577$ & $1,463 \pm 836$ & $0.004^{*}$ \\
\hline $\begin{array}{l}\text { Neutrophil-to-lymphocyte } \\
\text { ratio }\end{array}$ & $2.28 \pm 2.64$ & $3.13 \pm 7.62$ & $0.151^{*}$ \\
\hline $\begin{array}{l}\text { Monocyte-to-lymphocyte } \\
\text { ratio }\end{array}$ & $0.24 \pm 0.33$ & $0.55 \pm 2.09$ & $0.1^{*}$ \\
\hline uNGAL (ng/mL) & $23.1 \pm 41.8$ & $286.2 \pm 1,506.4$ & $<0.001^{*}$ \\
\hline Serum Cr (mg/dL) & $0.15 \pm 0.21$ & $0.15 \pm 0.17$ & $0.119^{*}$ \\
\hline BUN-to-Cr ratio & $36.9 \pm 13.6$ & $42.8 \pm 18.7$ & $0.015^{*}$ \\
\hline CRP (mg/L) & $27.8 \pm 37.0$ & $32.8 \pm 36.0$ & $0.564^{*}$ \\
\hline
\end{tabular}

The values are presented as mean \pm standard deviation or number (\%). Abbreviations: UTI, urinary tract infection; WBC, white blood cell; ANC, absolute neutrophil count; UNGAL, urinary neutrophil gelatinase-associated lipocalin; $\mathrm{Cr}$, creatinine; BUN, blood urea nitrogen; CRP, C-reactive protein.

*Mann-Whitney U test.

${ }^{\dagger}$ Chi-square test. 
WBC counts, monocyte counts, and UNGAL levels were significantly higher in the UTI group $(P<0.05)$ (Table 2$)$.

\section{Association between uNGAL and leukocyte differential counts}

Paired sample correlations were conducted by Spearman's correlation analysis with an adjustment for age, gender, and fever duration. In the UTI group, uNGAL levels showed positive correlations with ANC, monocyte counts, NLR, and MLR $(P<0.05)$. In the non-UTI group, uNGAL levels were associated only with the NLR $(P<0.05)$. In contrast, serum CRP concentrations were positively correlated with ANC, monocyte counts, NLR, and MLR in both the UTI and non-UTI groups $(P<0.05)$ (Table 3$)$.

Table 2. Age, Gender, Fever Duration-adjusted Predicted Means between the UTI and Non-UTI Groups

\begin{tabular}{lccc}
\hline & Non-UTI & UTI & P-value \\
\hline WBC count $\left(/ \mathrm{mm}^{3}\right)$ & $11,272 \pm 617$ & $13,436 \pm 490$ & $0.007^{*}$ \\
ANC $\left(/ \mathrm{mm}^{3}\right)$ & $7,169 \pm 411$ & $5,900 \pm 521$ & $0.06^{*}$ \\
Lymphocyte count $\left(/ \mathrm{mm}^{3}\right)$ & $4,590 \pm 203$ & $4,115 \pm 256$ & $0.153^{*}$ \\
Monocyte count $\left(/ \mathrm{mm}^{3}\right)$ & $1,013 \pm 82$ & $1,428 \pm 65$ & $<0.001^{*}$ \\
Neutrophil-to-lymphocyte ratio & $3.16 \pm 0.57$ & $2.19 \pm 0.72$ & $0.302^{*}$ \\
Monocyte-to-lymphocyte ratio & $0.53 \pm 0.15$ & $0.26 \pm 0.19$ & $0.277^{*}$ \\
UNGAL $(\mathrm{ng} / \mathrm{mL})$ & $26.6 \pm 136.8$ & $284.0 \pm 108.7$ & $<0.001^{*}$ \\
Serum Cr $(\mathrm{mg} / \mathrm{dL})$ & $0.16 \pm 0.02$ & $0.13 \pm 0.02$ & $0.317^{*}$ \\
BUN-to-Cr ratio & $42.3 \pm 1.6$ & $37.7 \pm 2.0$ & $0.074^{*}$ \\
CRP (mg/L) & $33.4 \pm 3.2$ & $26.7 \pm 4.2$ & $0.213^{*}$ \\
\hline
\end{tabular}

The values are presented as predicted mean \pm standard error.

Abbreviations: UTI, urinary tract infection; WBC, white blood cell; ANC, absolute neutrophil count; UNGAL, urinary neutrophil gelatinase-associated lipocalin; $\mathrm{Cr}$, creatinine; BUN, blood urea nitrogen; CRP, C-reactive protein.

*ANCOVA (analysis of covariance).

\section{Univariable and multivariable logistic regression analyses}

Univariable and multivariable logistic regression analyses were performed to test each parameter as an independent predictor of UTI. Univariable logistic regression analysis showed that age, female gender, WBC count, lymphocyte count, monocyte count, and UNGAL levels were significant predictors of UTI $(P<0.05)$. However, in a multivariable logistic regression analysis, only uNGAL levels independently predicted the presence of UTI ( $\mathrm{OR}=1.030$; 95\% CI: 1.016-1.044; $P<0.001$ ) (Table 4).

\section{ROC analyses}

The diagnostic properties of various markers were exa-

Table 3. Associations between uNGAL or Serum CRP and Leukocyte Differential Counts, Adjusted by Age, Gender, and Fever Duration in UTI and Non-UTI Groups

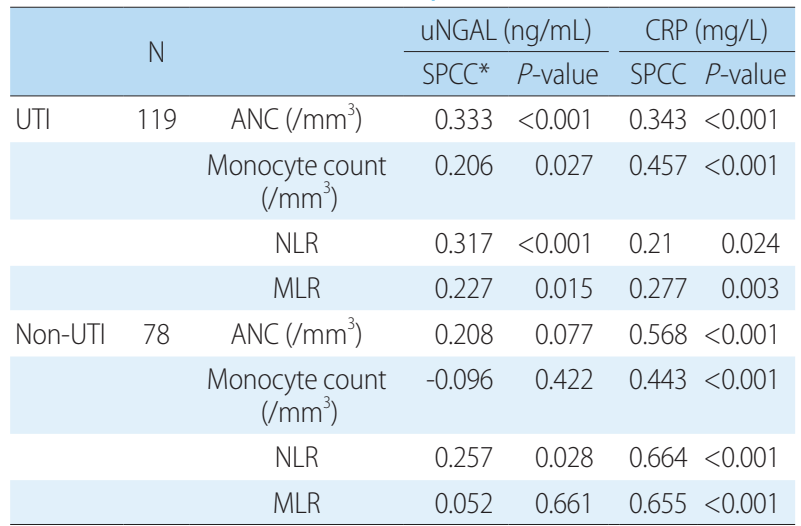

Abbreviations: UTI, urinary tract infection; UNGAL, urinary neutrophil gelatinase-associated lipocalin; CRP, C-reactive protein; ANC, absolute neutrophil count; NLR, neutrophil-to-lymphocyte ratio; $M L R$, monocyteto-lymphocyte ratio.

*Spearman's partial correlation coefficient.

Table 4. Univariable and Multivariable Logistic Regression Analysis for the UTI Group

\begin{tabular}{|c|c|c|c|c|}
\hline & \multicolumn{2}{|c|}{ Univariable logistic regression } & \multicolumn{2}{|c|}{ Multivariable logistic regression } \\
\hline & OR $(95 \% \mathrm{Cl})$ & $P$-value & OR $(95 \% \mathrm{Cl})$ & $P$-value \\
\hline Age (month) & 0.991 (0.984-0.999) & 0.031 & $1.002(0.991-1.013)$ & 0.76 \\
\hline Female gender & $0.485(0.271-0.867)$ & 0.015 & $0.743(0.347-1.594)$ & 0.446 \\
\hline WBC count $\left(/ \mathrm{mm}^{3}\right)$ & $1.000(1.000-1.000)$ & 0.003 & $0.999(0.997-1.001)$ & 0.575 \\
\hline ANC $\left(/ \mathrm{mm}^{3}\right)$ & $1.000(1.000-1.000)$ & 0.118 & $1.000(0.998-1.002)$ & 0.65 \\
\hline Lymphocyte count (/mm³) & $1.000(1.000-1.000)$ & 0.016 & 1.001 (0.999-1.003) & 0.555 \\
\hline Monocyte count $\left(/ \mathrm{mm}^{3}\right)$ & $1.000(1.000-1.000)$ & $<0.001$ & $1.002(1.000-1.004)$ & 0.109 \\
\hline Neutrophil-to-lymphocyte ratio & $1.033(0.961-1.110)$ & 0.383 & - & - \\
\hline Monocyte-to-lymphocyte ratio & $1.902(0.874-4.140)$ & 0.105 & $0.729(0.294-1.809)$ & 0.495 \\
\hline uNGAL (ng/mL) & $1.032(1.020-1.045)$ & $<0.001$ & $1.030(1.016-1.044)$ & $<0.001$ \\
\hline
\end{tabular}

Abbreviations: OR, odds ratio; Cl, confidence interval; WBC, white blood cell; ANC, absolute neutrophil count; uNGAL, urinary neutrophil gelatinase-associated lipocalin. 
mined for their predictability of UTI using ROC curves (Fig. 2). The area under the ROC curve was 0.89 for UNGAL (95\% CI: 0.824-0.939; $P=0.025), 0.7$ for monocyte count (95\% CI: 0.627-0.774; $P=0.038), 0.6$ for ANC (95\% CI: 0.518$0.687 ; P=0.043), 0.58$ for MLR (95\% CI: $0.500-0.663 ; P=$ 0.042 ), and 0.51 for NLR (95\% CI: $0.428-0.594 ; P=0.042$ ). The best cutoff value of uNGAL for detecting UTI was 34.8 $\mathrm{ng} / \mathrm{mL}$, with a sensitivity of $83.2 \%$ and a specificity of 84.6 $\%$, and that of monocyte counts was $932 / \mathrm{mm}^{3}$, with a sensitivity of $72.3 \%$ and a specificity of $60.3 \%$ (Table 5 ).

\section{Discussion}

In this study, we investigated the association of uNGAL levels with leukocyte differential counts in blood and the usefulness of leukocyte differential counts and UNGAL as

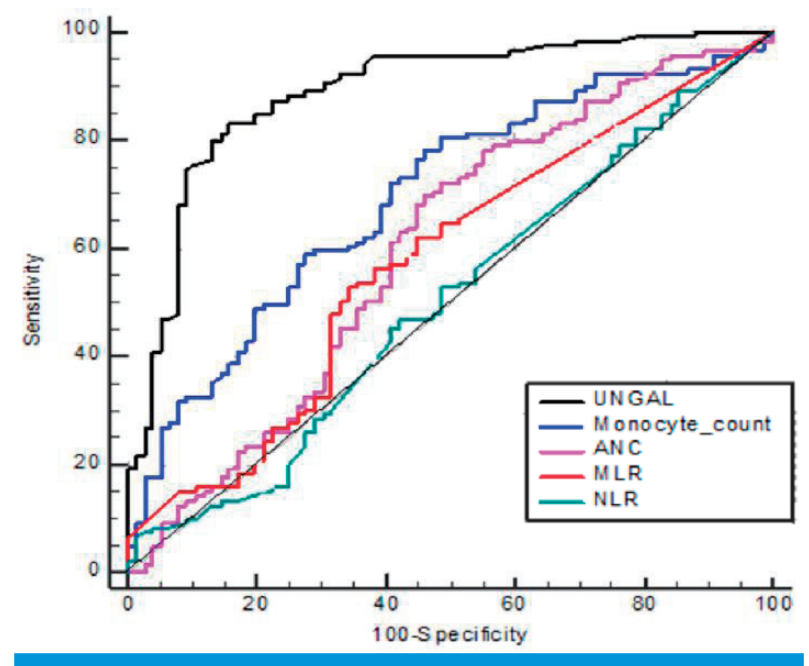

Fig. 2. Receiver operating characteristic curve of UNGAL levels, monocyte counts, the ANC, NLR, and MLR for predicting UTIs in children with fevers.

UNGAL, urinary urinary neutrophil gelatinase-associated lipocalin; ANC, absolute neutrophil count; NLR, neutrophil-to-lymphocyte ratio; MLR, monocyte-to-lymphocyte ratio. biomarkers of UTI in children with fever. While serum CRP was correlated with leukocyte differential counts in both children with and without UTIs, uNGAL levels were correlated with ANC, monocyte counts, and MLR only in children with febrile UTIs. Using univariable and multivariable logistic analyses, we showed that only uNGAL levels were an independent predictor of UTI. The AUCs of monocyte counts and UNGAL levels were considerably elevated for detecting UTI in children with suspected UTIs. These findings suggest that uNGAL levels could be related to leukocyte differential counts in the blood of children with febrile UTIs and monocyte counts as well as UNGAL levels may enhance the diagnostic accuracy for detecting UTIs in children with suspected UTIs.

Several studies have correlated leukocyte differential counts, especially neutrophil counts or the NLR, with bacterial infections ${ }^{13,25,26)}$. Also, lymphocytopenia has been reported to help predict bacterial infections ${ }^{27,28)}$. While significant research on monocyte counts or the MLR and infections are lacking, some studies have revealed a link between the MLR and bacterial infection. Naess et al. reported that the NLR and MLR were higher in the bacterial infection group, suggesting that they could be used for diagnosing bacterial infection ${ }^{13)}$. Also, Wang et al. recently found that the MLR is an important, independent factor in the diagnosis of severe Klebsiella pneumonia ${ }^{29)}$. Even compared to the NLR, the MLR appeared to be a better indicator for Klebsiella pneumonia evaluation, and also correlated with disease severity ${ }^{29)}$. In contrast, in critically ill and injured patients, the MLR was meaningful as a secondary marker for non-bacterial infections in patients with peritonitis ${ }^{30)}$. These results showed that the association between the MLR and bacterial infection has not been established. The link between monocytes and bacterial infection has been rarely studied, and, to the best of our knowledge, there has been no clinical research on the cli-

Table 5. Predictive Value of Biomarkers for UTI using the ROC Curve

\begin{tabular}{lllcccc}
\hline & AUC & $95 \% \mathrm{Cl}$ & P-value & Cutoff value & Sensitivity (\%) & Specificity (\%) \\
\hline uNGAL $(\mathrm{ng} / \mathrm{mL})$ & 0.89 & $0.824-0.939$ & 0.025 & 34.8 & 83.2 & 84.6 \\
Monocyte count $\left(/ \mathrm{mm}^{3}\right)$ & 0.7 & $0.627-0.774$ & 0.038 & 932 & 72.3 & 60.3 \\
ANC $\left(/ \mathrm{mm}^{3}\right)$ & 0.6 & $0.518-0.687$ & 0.043 & 4.572 & 69.8 & 54 \\
MLR & 0.58 & $0.500-0.663$ & 0.042 & 0.21 & 52.9 & 66.7 \\
NLR & 0.51 & $0.428-0.594$ & 0.042 & 1.51 & 16 & 75.6 \\
\hline
\end{tabular}

Abbreviations: AUC, area under the curve; Cl, confidence interval; uNGAL, urinary neutrophil gelatinase-associated lipocalin; NLR, neutrophil-to-lymphocyte ratio; MLR, monocyte-to-lymphocyte ratio; ANC, absolute neutrophil count. 
nical utility of monocyte counts in the evaluation of UTI.

When an infection occurs, neutrophils and monocytes move to the peripheral site, which is essential for defending against pathogens ${ }^{18)}$. As the infection progresses, the number of neutrophils and monocytes developing in the bone marrow increases rapidly and the cells migrate to the inflamed tissue ${ }^{18}$. CCL2/CCR2-dependent inflammatory monocytes are released from the bone marrow. Eventually, these monocytes migrate to tissues and differentiate into macrophages or dendritic cells ${ }^{31)}$. Monocytes are also involved in tissue healing, the removal of pathogens and dead cells, and the onset of adaptive immunity ${ }^{20}$. Macrophages differentiated from monocytes are under the mucous membrane of the urinary tract, and, in the case of infection, more macrophages are recruited to this site ${ }^{211}$. When activated due to infection, macrophages produce important cytokines and chemokines that regulate their activity. In addition, other nearby immune cells are also activated, affecting the inflammatory response ${ }^{32}$. In the present study, monocyte counts in addition to uNGAL levels were higher in the UTI group compared to the non-UTI group, even after adjustment for age, gender, and fever duration. The ANC, NLR, and MLR were not different between patients with UTI and non-UTI. In the UTI group, there was a correlation between uNGAL levels and ANC, monocyte counts, and the MLR. The NLR showed a positive relationship with uNGAL levels both in the UTI and non-UTI groups. In contrast, there was no relationship between uNGAL levels and monocyte counts or the MLR in the non-UTI group. Therefore, monocyte counts seem to be more specific than any other leukocyte differential count in the detection of UTIs in this clinical setting.

NGAL, which is highly expressed in monocytes/macrophages as well as neutrophils, serves as a mediator of the innate immune response ${ }^{9,333}$. As an iron carrier protein, it plays a significant role in limiting bacterial growth by binding bacterial siderophores ${ }^{9}$. Many studies including ours have shown that uNGAL measurements could be helpful in the prediction of UTIs in children ${ }^{11,34,35)}$. Previously, we identified the uNGAL and uNGAL/Cr were promising evaluations to diagnose and manage febrile UTIs in children. The AUC for detecting a UTI in children was 0.89 for uNGAL levels and 0.9 for uNGAL/Cr ${ }^{34)}$. Lubell et al. demonstrated that in a total of 260 children with suspected
UTIs, uNGAL had higher sensitivity than Gram stains, the combination of leukocyte esterase activity, or nitrite positivity for the detection of $\mathrm{UTI}^{11)}$. The AUC for detecting a UTI was 0.871 for Gram stain, with a sensitivity of $74.3 \%$ and a specificity of $100 \%{ }^{11)}$. The AUC was 0.858 for nitrite positivity, with a sensitivity of $20.0 \%$ and a specificity of 100 $\%^{11)}$. At a cutoff level of $39.1 \mathrm{ng} / \mathrm{mL}$, the AUC for uNGAL was 0.978 , with a sensitivity of $97.1 \%$ and a specificity of $95.6 \%{ }^{11)}$. Jagadesan et al. also reported that uNGAL levels could be more sensitive and specific than urine WBCs in identifying $\mathrm{UTI}^{35}{ }^{35}$. The AUC for detecting a UTI was 0.70 for urine WBCs, with a sensitivity of $70.6 \%$ and a specificity of $53 \%{ }^{35)}$. uNGAL showed higher AUC value of 0.76 , with a sensitivity of $79.4 \%$ and a specificity of $68.2 \%{ }^{35)}$. Consistent with these findings, we also confirmed that UNGAL levels had a high discriminating ability for UTIs in children with suspected UTIs. The AUC to predict UTI was 0.89 for uNGAL, with the best cutoff value of $34.8 \mathrm{ng} / \mathrm{mL}$ (sensitivity $83.2 \%$, specificity $84.6 \%$ ). Additionally, in the ROC analysis, monocyte counts were helpful for detecting UTIs. At a cutoff monocyte count of $932 / \mathrm{mm}^{3}$, the AUC for identifying UTI was 0.70 (sensitivity $72.3 \%$, specificity $60.3 \%$ ). These findings suggest that elevated uNGAL levels in children with UTIs may be associated with increased monocyte counts in blood. And the monocyte counts, as well as uNGAL levels, may be helpful in detecting UTIs in children with suspected UTIs. However, in a multivariable logistic regression analysis, only uNGAL levels independently predicted the presence of a UTI. Serum monocyte counts were not retained as a predictor of UTI in children with fevers. Although monocyte counts are not as high in AUC, sensitivity and specificity as UNGAL, it was confirmed that monocyte counts were related to UNGAL in the UTI group. In addition, monocyte counts are highly effective as a simple blood test routinely performed in patients.

CRP is an inflammatory marker long used as an indicator of acute infection ${ }^{36-38)}$. CRP and WBC counts, including the ANC, have been reported to increase in acute bacterial infections and positively correlate with each other ${ }^{38)}$. However, in our clinical setting, CRP did not seem to be specific in predicting UTIs in children with suspected UTIs. Serum CRP showed positive correlations with the ANC, monocyte count, NLR, and MLR in both the UTI and nonUTI groups. Further, the CRP levels were not different in 
children with or without UTI, even after adjustment for age, gender, and fever duration. Therefore, the usefulness of serum CRP may be limited for predicting UTI in children with fevers.

This study had some limitations. First, the sample size was relatively small because it was a single-center retrospective study. Multicenter, prospective studies involving a large number of patients are needed to overcome these limitations. Second, we could not completely confirm the viral cause of illness in the non-UTI group since we did not perform viral cultures in all children in the non-UTI group. Third, we only classified children with fever into UTI group and non-UTI group, and there was no control group without fever. Fourth, only uNGAL was analyzed in this study. Since plasma NGAL is a more characteristic marker for systemic inflammatory conditions ${ }^{39)}$, studies are needed to compare the relationship between plasma NGAL levels and leukocyte differential counts.

In conclusion, uNGAL levels may be associated with blood leukocyte differential counts in children with febrile UTIs. Both uNGAL measurements and monocyte counts can help in identifying UTI in a child with a suspected UTI, although uNGAL seems to be most valuable in predicting UTI. Further studies involving a large number of patients are needed to clarify the clinical significance of leukocyte differential counts, especially monocyte in children with suspected UTIs.

\section{Conflicts of interest}

No potential conflict of interest relevant to this article was reported.

\section{Patient consent}

This study was approved by the Institutional Review Board (IRB), and the consent was waived due to the nature of the retrospective study [IRB number 2019GR0237].

\section{ORCID IDs}

Ji Won Jang https://orcid.org/0000-0003-4959-2396

Hyung Eun Yim https://orcid.org/0000-0001-9805-9278

Kee Hwan Yoo https://orcid.org/0000-0001-6490-4293

\section{References}

1. Lee SJ. Clinical guideline for childhood urinary tract infection (second revision). Child Kidney Dis 2015;19:56-64.

2. Strohmeier Y, Hodson EM, Willis NS, Webster AC, Craig JC. Antibiotics for acute pyelonephritis in children. Cochrane Database Syst Rev, 2014;28;CD003772.

3. National Collaborating Centre for Women's and Children's Health. Urinary Tract Infection in Children: Diagnosis, Treatment and Long Term Management. London, England: RCOG Press; 2007.

4. Han SY, Lee IR, Park SJ, Kim JH, Shin JI. Usefulness of neutrophillymphocyte ratio in young children with febrile urinary tract infection. Korean J Pediatr 2016;59:139-44.

5. Tullus K. Difficulties in diagnosing urinary tract infections in small children. Pediatr Nephrol 2011;26:1923-6.

6. Tullus K. Low urinary bacterial counts: do they count? Pediatr Nephrol 2016;31:171-4.

7. Masajtis-Zagajewska A, Nowicki M. New markers of urinary tract infection. Clinica Chimica Acta 2017;471:286-91.

8. Yim HE. Neutrophil gelatinase-associated lipocalin and kidney diseases. Child Kidney Dis 2015;19:79-88.

9. Ichino M, Kuroyanagi Y, Kusaka M, Mori T, Ishikawa K, Shiroki R, et al. Increased urinary neutrophil gelatinase associated lipocalin levels in a rat model of upper urinary tract infection. J Urol 2009; 181:2326-31.

10. Fjaertoft G, Foucard T, Xu S, Venge P. Human neutrophil lipocalin $(\mathrm{HNL})$ as a diagnostic tool in children with acute infections: a study of the kinetics. Acta Paediatr 2005;94:661-6.

11. Lubell TR, Barasch JM, Xu K, leni M, Cabrera KI, Dayan PS. Urinary neutrophil gelatinase-associated lipocalin for the diagnosis of urinary tract infections. Pediatrics 2017;140:e20171090.

12. Skowron B, Baranowska A, Dobrek L, Ciesielczyk K, Kaszuba-Zwoinska J, Wiecek G, et al. Urinary neutrophil gelatinase-associated lipocalin, kidney injury molecule-1, uromodulin, and cystatin C concentrations in an experimental rat model of ascending acute kidney injury induced by pyelonephritis. J Physiol Pharmacol 2018;69:625-37.

13. Naess A, Nilssen SS, Mo R, Eide GE, Sjursen H. Role of neutrophil to lymphocyte and monocyte to lymphocyte ratios in the diagnosis of bacterial infection in patients with fever. Infection 2017;45:299-307.

14. Loonen AJ, de Jager CP, Tosserams J, Kusters R, Hilbink M, Wever $P C$, et al. Biomarkers and molecular analysis to improve blood- 
stream infection diagnostics in an emergency care unit. PloS One 2014;9:e87315.

15. Riley LK, Rupert J. Evaluation of patients with leukocytosis. AM Fam Phys 2015;92:1004-11.

16. Terradas R, Grau S, Blanch J, Riu M, Saballs P, Castells X, et al. Eosinophil count and neutrophil-lymphocyte count ratio as prognostic markers in patients with bacteremia: a retrospective cohort study. PloS One 2012;7:e42860.

17. Meshaal MS, Nagi A, Eldamaty A, Gaber M, Rizk HJTEHJ. Neutrophil-to-lymphocyte ratio (NLR) and platelet-to-lymphocyte ratio $(P L R)$ as independent predictors of outcome in infective endocarditis (IE). Egypt Heart J 2019;71:13.

18. Serbina NV, Hohl TM, Cherny M, Pamer EG. Selective expansion of the monocytic lineage directed by bacterial infection. J Immunol 2009;183:1900-10.

19. Dixit A, Bottek J, Beerlage AL, Schuettpelz J, Thiebes S, Brenzel A, et al. Frontline science: proliferation of Ly6C(+) monocytes during urinary tract infections is regulated by IL-6 trans-signaling. J Leukoc Biol 2018;103:13-22.

20. Ingersoll MA, Platt AM, Potteaux S, Randolph GJ. Monocyte trafficking in acute and chronic inflammation. Trends Immunol 2011; 32:470-7.

21. Abraham SN, Miao Y. The nature of immune responses to urinary tract infections. Nat Rev Immunol 2015;15:655-63.

22. Bieber K, Autenrieth SE. Insights how monocytes and dendritic cells contribute and regulate immune defense against microbial pathogens. Immunobiology 2015;220:215-26.

23. Pérez RP, Ortega MJC, Álvarez JA, Baquero-Artigao F, Rico JCS, Zúñiga RV, et al. Recommendations on the diagnosis and treatment of urinary tract infection]. Anales de Pediatría 2019;90:400. e1-9.

24. Krzeminska E, Wyczalkowska-Tomasik A, Korytowska N, Paczek L. Paczek comparison of two methods for determination of NGAL levels in urine: ELISA and CMIA. J Clin Lab Anal 2016;30:956-60.

25. Lowsby R, Gomes C, Jarman I, Lisboa P, Nee PA, Vardhan M, et al. Neutrophil to lymphocyte count ratio as an early indicator of blood stream infection in the emergency department. Emerg Med J 2015:32:531-4.

26. Turak O, Özcan F, İ̧leyen A, Başar FN, Gül M, Yilmaz S, et al. Usefulness of neutrophil-to-lymphocyte ratio to predict in-hospital outcomes in infective endocarditis. Can J Cardiol 2013;29:1672-8.

27. de Jager CP, van Wijk PTL, Mathoera RB, de Jongh-Leuvenink J, van der Poll T, Wever PC. Lymphocytopenia and neutrophillymphocyte count ratio predict bacteremia better than conven- tional infection markers in an emergency care unit. Crit Care 2010;14:R192-R20021034463.

28. Wyllie DH, Bowler ICJW, Peto TEA. Relation between lymphopenia and bacteraemia in UK adults with medical emergencies. J Clin Pathol 2004:57:950-5.

29. Wang Jl, Lu Xy, Xu Xh, Zhang Kj, Gong H, Lv D, et al. Predictive role of monocyte-to-lymphocyte ratio in patients with Klebsiella pneumonia infection: a single-center experience. Medicine (Baltimore) 2019;98:e17215.

30. Djordjevic D, Rondovic G, Surbatovic M, Stanojevic I, Udovicic I, Andjelic T, et al. Neutrophil-to-lymphocyte ratio, monocyte-tolymphocyte ratio, platelet-to-lymphocyte ratio, and mean platelet volume-to-platelet count ratio as biomarkers in critically ill and injured patients: which ratio to choose to predict outcome and nature of bacteremia?. Mediators Inflamm 2018;15:2018:3758068.

31. Serbina NV, Pamer EG. Monocyte emigration from bone marrow during bacterial infection requires signals mediated by chemokine receptor CCR2. Nature Immunol 2006;7:311-7..

32. Duell BL, Carey AJ, Dando SJ, Schembri MA, Ulett GC. Human bladder uroepithelial cells synergize with monocytes to promote IL-10 synthesis and other cytokine responses to uropathogenic Escherichia coli. PLOS ONE 2013;8:e78013.

33. Cai L, Rubin J, Han W, Venge P, Xu S. The origin of multiple molecular forms in urine of HNL/NGAL. Clin J Am Soc Nephrol 2010;5: 2229-35.

34. Yim HE, Yim H, Bae ES, Woo SU, Yoo KH. Predictive value of urinary and serum biomarkers in young children with febrile urinary tract infections. Pediatr Nephrol 2014;29:2181-9.

35. Jagadesan I, Agarwal I, Chaturvedi S, Jose A, Sahni RD, Fleming JJ. Urinary neutrophil gelatinase associated lipocalin - a sensitive marker for urinary tract infection in children. Indian J Nephrol 2019;29:340-4.

36. Zarkesh M, Sedaghat F, Heidarzadeh A, Tabrizi M, Moghadam KB, Ghesmati S. Diagnostic Value of IL-6, CRP, WBC, and Absolute Neutrophil Count to Predict Serious Bacterial Infection in Febrile Infants. Acta Medica Iranica 2015;53.

37. Sproston NR, Ashworth JJ. Ashworth Role of C-reactive protein at sites of inflammation and infection. Front Immunol 2018;9:754.

38. Shaikh S, Salim E, Ram PV, Memon SS, Zubairi A, Khawaja SA, et al. Correlation of C-reactive protein and total leukocyte count in acute infections. Pak J Surg 2019;35:271-4.

39. Forster CS, Devarajan P. Neutrophil gelatinase-associated lipocalin: utility in urologic conditions. Pediatric Nephrology 2017;32: 377-81. 